Kazunari Hayashida

Nagoya Math. J.

Vol. 36 (1969), 99-115

\title{
ON BOUNDARY VALUE PROBLEMS FOR ELLIPTIC EQUATIONS IN A SINGULAR DOMAIN
}

\author{
KAZUNARI HAYASHIDA
}

1. Let $\Omega$ be a bounded domain in the plane and denotes its closure and boundary by $\bar{\Omega}$ and $\partial \Omega$, respectively. We shall say that the domain $\Omega$ is regular, if every point $P \in \partial \Omega$ has an 2-dimensional neighborhood $U$ such that $\partial \Omega \cap U$ can be mapped in a one-to-one way onto a portion of the tangent line through $P$ by a mapping $T$ which together with its inverse is infinitely differentiable. Let $L$ be an elliptic operator of order $2 m$ defined in $\bar{\Omega}$ and let $\left\{B_{j}\right\}_{j=1}^{m}$ be a normal set of boundary operators of orders $m_{j}<2 m$. If $f$ is a given function in $\Omega$, the boundary value problem $\Pi\left(L, f, B_{j}\right)$ will be to find a solution $u$ of

$$
L u=f \text { in } \Omega
$$

satisfying

$$
B_{j} u=0 \quad \text { on } \partial \Omega, \quad j=1, \cdots, m \text {. }
$$

Schechter [8] proved the following: If the set $\left\{B_{j}\right\}_{j=1}^{m}$ is normal and covers $L$, there is another normal set $\left\{B_{j}^{\prime}\right\}_{j=1}^{m}$ such that a solution of the problem $\Pi\left(L, f, B_{j}\right)$ exists if and only if the only solution of $\Pi\left(L^{*}, 0, B_{j}^{\prime}\right)$ is $u=0$. Here $L^{*}$ denotes the formal adjoint of $L$.

We consider the problem $\Pi\left(L, f, B_{j}\right)$ when $\Omega$ is not regular in our sense. When $\Omega$ is a domain in the plane, we shall call it singular if $\partial \Omega$ consists of a set $\left\{\Gamma_{i}\right\}_{i=1}^{N}$ of boundary portions which are sufficiently smooth and satisfy the following conditions.

(i) Each boundary portion $\Gamma_{i}$ is a slit in $\bar{\Omega}$ or is contained in the outer boundary of $\Omega$. When $\Gamma_{i}$ is a slit, we distinguish between both sides.

(ii) If $\Gamma_{i}$ and $\Gamma_{i^{\prime}}$ are contained in the outer boundary and adjoining at

Received September 17, 1968. 
$S$, they are tangent at $S$ of infinite order from the interior. More precisely, some neighborhood of $S$ in $\Omega$ can be mapped in a one-to-one $C^{\infty}$ way into an open disk which has an incision.

In this note we consider general boundary value problems for elliptic partial differential equations when $\Omega$ is singular in our sense.

Let $\left\{B_{i j}\right\}_{j=1}^{m}$ be a set of partial differential operators on each $\Gamma_{i}$. The problem we consider is the following: Given a function $f$ in $\Omega$, find the solution $u$ such that

$$
\begin{gathered}
L u=f \text { in } \Omega, \\
B_{i j} u=0 \text { on } \Gamma_{i}, \\
i=1, \cdots, N, \quad j=1, \cdots, m .
\end{gathered}
$$

Our method employs coerceiveness inequalities specially adapted to the probelm. In neighborhood of points of the inner part of $\Gamma_{i}$, no new inequalities are needed (c.f. $[1,8]$ ). For the endpoint of $\Gamma_{i}$ we obtain special inequalities which are reduced to the mixed boundary value problems.

Mixed boundary value problems in a planar domain were studied quite extensively by Peetre [7] and Shamir [12]. They used some properties of the Hilbert transform on the half line which were given in [5], [11], and [15]. For arbitrary dimension, Schechter [9] treated the mixed boundary problems under a rather complicated compatibility condition. In this note our proof relies upon mainly the results of Schechter [9] and Shamir [12].

2. Let $R^{n}$ be the $n$-dimensional Euclidean space. Throughout this note we consider only the case $n=1$ or 2 . Points in $R^{2}$ are denoted by $P=(x, t)$ and $|P|^{2}=|x|^{2}+|t|^{2}$. The half space $t>0 \quad(<0)$ is denoted by $R_{+}^{2}\left(R_{-}^{2}\right)$. Let $\alpha=\left(\alpha_{1}, \alpha_{2}\right)$ be a multi-index of non-negative integers with length $|\alpha|=\alpha_{1}+\alpha_{2}$. We shall write

$$
D=\left(D_{x}, D_{t}\right), \quad D^{\alpha}=D_{x}^{\alpha_{1}} D_{t}^{\alpha_{2}} \quad\left(D_{x}=\partial / \partial x, D_{t}=\partial / \partial t\right) .
$$

We consider an elliptic differential operator of the form

$$
L(D)=\sum_{|\alpha|=2 m} a_{\aleph} D^{\alpha}
$$

where the coefficients $a_{\star}$ are complex numbers and $2 m$ is the order of $L(D)$. The characteristic polynomial corresponding to $L(D)$ is

$$
L(\xi, \eta)=\sum_{|\alpha|=2 m} a_{\lrcorner} \xi^{x_{1} \eta^{\alpha}}
$$

We set 


$$
L_{1}(\xi, \tau)=L(\xi, \tau), \quad L_{2}(\xi, \tau)=L(\xi,-\tau)
$$

and

$$
B_{1 j}^{-}(D)=D_{t}^{j-1}, \quad B_{2 j}^{-}(D)=(-1)^{j} D_{t}^{j-1}, \quad j=1, \cdots, 2 m .
$$

In this section we shall mainly describe Agmon-Douglis-Nirenberg's results for the boundary value problem of the elliptic system:

$$
\begin{aligned}
& L_{1}(D) u_{1}=f_{1}, \quad L_{2}(D)=f_{2}, \quad t>0, \\
& B_{1 j}^{-} u_{1}+B_{2 j}^{-} u_{2}=\varphi_{j}, \quad t=0, \quad j=1, \cdots, 2 m .
\end{aligned}
$$

Denote by $\tau_{i, k}^{+}(\xi)$ (or $\left.\tau_{\bar{i}, k}^{-}(\xi)\right), k=1, \cdots, m$ the roots of $L_{i}(\xi, \tau)=0$ with positive (or negative) imaginary parts, and set

$$
\begin{aligned}
& L_{i}^{ \pm}(\xi, \tau)=\prod_{1}^{m}\left(\tau-\tau_{i, k}^{ \pm}(\xi)\right), \\
& M^{+}(\xi, \tau)=L_{1}^{+}(\xi, \tau) L_{2}^{+}(\xi, \tau), \quad \xi \neq 0 .
\end{aligned}
$$

Then we have

Lemma 2. 1. The boundary value problem (2.4) satisfies the Complementing Condition in the sense of [2]. That is, for each real $\xi \neq 0$ the relations

$$
\sum_{j=1}^{2 m} \lambda_{j} B_{1 j}^{-}(\tau) L_{2}(\xi, \tau)=U_{1}(\tau) M^{+}(\xi, \tau)
$$

$$
\sum_{j=1}^{2 m} \lambda_{j} B_{2 j}^{-}(\tau) L_{1}(\xi, \tau)=U_{2}(\tau) M^{+}(\xi, \tau)
$$

imply that $U_{1}(\tau), U_{2}(\tau)$ and the $\lambda_{j}$ all vanish, where the $\lambda_{j}$ are complex constants and the $U_{i}(\tau)$ are polynomials.

Proof. We note that (2.5) are equivalent to

$$
\begin{aligned}
& \sum_{j=1}^{2 m} \lambda_{j} B_{1 j}^{-}(\tau)=U_{1}^{\prime}(\tau) L_{1}^{+}(\xi, \tau), \\
& \sum_{j=1}^{2 m} \lambda_{j} B_{2 j}^{-}(\tau)=U_{2}^{\prime}(\tau) L_{2}^{+}(\xi, \tau),
\end{aligned}
$$

where $U_{i}^{\prime}(\tau)$ are other polynomials. From (2.2) we have $L_{2}^{+}(\xi, \tau)=L^{+}(-\xi, \tau)$. Hence the relations (2.6) imply that

$$
\begin{aligned}
& \sum_{j=1}^{2 m} \lambda_{j} \tau^{j-1}=U_{1}^{\prime}(\tau) L^{+}(\xi, \tau), \\
& \sum_{j=1}^{2 m} \lambda_{j}(-1)^{j} \tau^{j-1}=U_{2}^{\prime}(\tau) L^{+}(-\xi, \tau) .
\end{aligned}
$$


Thus it follows that

$$
-U_{1}^{\prime}(-\tau) L^{+}(\xi,-\tau)=U_{2}^{\prime}(\tau) L^{+}(-\xi, \tau) .
$$

Noting that $L^{+}(\xi,-\tau)=(-1)^{m} L^{-}(-\xi, \tau)$, we see

$$
(-1)^{m-1} U_{1}^{\prime}(-\tau) L^{-}(-\xi, \tau)=U_{2}^{\prime}(\tau) L^{+}(-\xi, \tau) \text {. }
$$

Since $U_{i}^{\prime}(\tau)$ are of degree at most $m-1$, the relation (2.7) means that every $U_{i}^{\prime}(\tau)$ vanishes. Hence all $\lambda_{j}$ vanish. This completes the proof.

We first consider the problem (2.4) in the case $f_{1}=f_{2}=0$ and $\varphi_{1}(x)$, $\varphi_{2}(x) \in C_{0}^{\infty}(R)^{1)}$. This problem can be solved by the formula

$$
u_{i}(x, t)=\sum_{j=1}^{2 m} \int K_{i j}(x-y, t) \varphi_{j}(y) d y, \quad i=1,2,
$$

where $K_{i j}(x, t)$ are Poisson kernels of class $C^{\infty}$ for $t>0$ except at the origin. We set

$$
G(z, M)=-(2 \pi i M)^{-1} z^{M}\left(\log (z / i)-\sum_{k=1}^{M} 1 / k\right)
$$

Then we have for odd $q>0$

$$
\begin{gathered}
K_{i j}(x, t)=\left(\frac{\partial}{\partial x}\right)^{(1+q) / 2} \sum_{ \pm}\left( \pm \frac{\partial}{\partial x}\right)^{2 m-1-m \bar{j}} R_{i j}(x, t ; \pm 1) \\
\left(m_{j}^{-}=\operatorname{deg} . B_{j}^{-}=j-1\right)
\end{gathered}
$$

and

$$
\begin{aligned}
R_{i j}(x, t, \pm 1) & =(2 \pi i)^{-1} \sum_{ \pm} \int_{\tau} L_{i}( \pm 1, \tau) \times \\
& \times G( \pm x+t \tau, q+2 m-1) \\
& \times \sum_{l=0}^{2 m-1} c_{i \imath}^{ \pm} \frac{M_{2 m-l-1}( \pm 1, \tau)}{M^{+}( \pm 1, \tau)} d \tau,
\end{aligned}
$$

where $\gamma$ is a closed curve in $\operatorname{Im} \tau>0$ enclosing all the zeros of $M^{+}( \pm 1, \tau)$ and $c_{i l j}^{ \pm}$are constants depending on $L_{1}$ and $L_{2}$.

The functions $M_{2 m-l-1}$ in (2. 10) are polynomials such that

$$
\begin{array}{r}
(2 \pi i)^{-1} \int_{\tau} \frac{M_{2 m-1-l}( \pm 1, \tau)}{M( \pm 1, \tau)} \tau^{k} d \tau=\delta_{l k}, \\
0 \leqq j, k \leqq 2 m-1 .
\end{array}
$$

1) We denote $R^{1}$ by $R$. 
It is seen that $K_{i j}(x, t)$ are of class $C^{\infty}$ for $t \geqq 0$, except at the origin, and satisfy

$$
\left|D^{\alpha} K_{i j}\right| \leqq C\left(x^{2}+t^{2}\right)^{\left(m_{j}^{-}-|\alpha|-1\right) / 2}\left(1+\left|\log \left(x^{2}+t^{2}\right)\right|\right) .
$$

We now consider the problem (2. 4) for $f_{1}, f_{2} \in C_{0}^{\infty}\left(\bar{R}_{+}^{2}\right)$. For this purpose we extend $f_{i}$ to the whole plane $R^{2}$ as functions with compact support of class $C^{N}$ (see $\left[1\right.$, p. 519]). Let $f_{i}^{(N)}(x, t)$ be the extended functions. Having chosen some large $N$, we set

$$
v_{i}(P)=\int \Gamma_{i}(P-Q) f_{i}^{(N)}(Q) d Q
$$

where $\Gamma_{i}(P)$ is a fundamental solution of the equation $L_{i} u=0$. The function $v_{i}$ satisfies $L_{i} v_{i}=f_{i}^{(N)}$ and it is known that

$$
D^{\alpha} v_{i}(P)=O\left(|P|^{2 m-2-|\alpha|}(1+|\log | P \|), \quad P \rightarrow \infty .\right.
$$

In addition, we see that for $\beta$ such that $|\beta|=2 m$

$$
D^{\beta} v_{i}=\int D^{\beta} \Gamma(P-Q) f_{i}^{(N)}(Q) d Q
$$

and that $D^{\beta} \Gamma$ is a homogeneous kernel of degree -2 to which CalderonZygmund's results on singular integrals can be applied.

Proposition 2. $1^{1)}$. Let $u_{i}$ be $C^{\infty}$ solutions with compact support in $t \geqq 0$ of the problem (2. 4). Then it holds

$$
\begin{gathered}
D^{\alpha} u_{i}=D^{\alpha} v_{i}+\sum_{j=1}^{2 m} \int D^{\alpha} K_{i j}(x-y, t) \cdot\left(\varphi_{j}(y)-\psi_{j}(y)\right) d y, \\
(|\alpha| \geqq 2 m-1)
\end{gathered}
$$

where $\psi_{j}(y)=B_{1 j}^{-} v_{1}(y, 0)+B_{2 j}^{-} v_{2}(y, 0)$.

This was proved in detail in [1] and [2] for $|\alpha| \geqq 2 m$ and we easily verify it for $|\alpha|=2 m-1$.

For an integral $r \geqq 0$ we use the norm

$$
\|u, \Omega\|_{r}=\sum_{|\alpha| \leqq r}\left(\int_{\Omega}\left|D^{\alpha} u\right|^{2} d x\right)^{1 / 2},
$$

where $\Omega=R^{n}$ or $R_{+}^{n}(n=1,2)$. For a real $s \geqq 0$ we define the seminorms

1) For single equations this was verified in [12]. 


$$
\begin{aligned}
& {[u, \Omega]_{s}=\left(\int_{\Omega} \int_{\Omega} \frac{|u(x)-u(y)|^{2}}{|x-y|^{n+2 s}} d x d y\right)^{1 / 2}, \quad 0<s<1,} \\
& {[u, \Omega]_{s}=\sum_{|\alpha|=s}\left[D^{\alpha} u, \Omega\right]_{s-[s]}, \quad 1 \leqq s .}
\end{aligned}
$$

Let $W^{s}(\Omega)$ be the completion of $C^{\infty}(\bar{\Omega})$ with respect to the norm

$$
\|u, \Omega\|_{s}=\|u, \Omega\|_{[s]}+[u, \Omega]_{s} .
$$

Then we have from (2. 14) (c.f. [1])

$$
\left\|v_{i}, R_{+}^{2}\right\|_{2 m} \leqq C\left\|f_{i}^{(N)}, R^{2}\right\|_{0} \leqq C\left\|L_{j} u_{i}, R_{+}^{2}\right\|_{0} .
$$

Proposition 2.2. (c.f. [2]) Assume that $u_{i}(x, t)$ belong to $C_{0}^{\infty}\left(\bar{R}_{+}^{2}\right)$ and $l \geqq 2 m$. Then there is a constant $C$ such that

$$
\begin{gathered}
\left\|u_{1}, R_{+}^{2}\right\|_{l}+\left\|u_{2}, R_{+}^{2}\right\|_{l} \leqq C\left(\left\|L_{1} u_{1}, R_{+}^{2}\right\|_{l-2 m}\right. \\
+\left\|L_{2} u_{2}, R_{+}^{2}\right\|_{l-2 m}+\sum_{j=1}^{2 m}\left\|B_{1 j}^{-} u_{1}+B_{2 j}^{-} u_{2}, R\right\|_{l-m-j^{-\frac{1}{2}}} \\
\left.+\left\|u_{1}, R_{+}^{2}\right\|_{0}+\left\|u_{2}, R_{+}^{2}\right\|_{0}\right) .
\end{gathered}
$$

This was proved in [1],[2] under potential theoretic considerations.

3. Let $\left\{B_{j}^{+}\right\}_{j=1}^{m}$ be a set of boundary operators with constant coefficients. We assume that $B_{j}^{+}$is homogeneous of degree $m_{j}^{+}(<2 m)$ and that the Complementing Condition on $\left\{B_{j}^{+}\right\}$is satisfied. In this section we shall give a proof of the following mixed a priori estimates for $u_{i} \in C_{0}^{\infty}\left(\bar{R}_{+}^{2}\right)$,

$$
\begin{aligned}
\left\|u_{1}, R_{+}^{2}\right\|_{2 m} & +\left\|u_{2}, R_{+}^{2}\right\|_{2 m} \leqq C\left(\left\|L_{1} u_{1}, R_{+}^{2}\right\|_{0}+\left\|L_{2} u_{2}, R_{+}^{2}\right\|_{0}\right. \\
& +\sum_{j=1}^{2 m}\left\|B_{1 j}^{-} u_{1}+B_{2 j}^{-} u_{2}, R_{-}\right\|_{2 m-m-j} \\
& +\sum_{j=1}^{2 m}\left\|B_{1 j}^{+} u_{1}+B_{2 j}^{+} u_{2}, R_{+}\right\|_{2 m-m_{j}^{+}} \\
& \left.+\left\|u_{1}, R_{+}^{2}\right\|_{0}+\left\|u_{2}, R_{+}^{2}\right\|_{0}\right) .
\end{aligned}
$$

The proof of (3. 1) is obtained in a similar manner to the method developed by Shamir for single equations (c.f. [11]).

We consider now the Hilbert transform on $R$ defined by

$$
\left(\mathscr{H}^{ \pm} f\right)(x)=\lim _{\varepsilon \downarrow 0}(2 \pi i)^{-1} \int_{-\infty}^{\infty} \frac{f(y)}{x+i \varepsilon-y} d y .
$$

Put $\mathscr{A} \varphi=\left(C \mathscr{H}^{+}+D \mathscr{H}^{-}\right) \varphi$, where $\varphi$ is a $2 m$ dimensional vector function and $C$ and $D$ are $2 m \times 2 m$ matrices with constant coefficients. 
Proposition 3.1. If $C$ and $D$ are non singular and if the eigenvalues of $C^{-1} D$ do not lie on the negative real axis, then for $\phi \in W^{\frac{1}{2}}(R)$

$$
[\phi, R]_{\frac{1}{2}} \leqq C\left(\left[\phi, R_{-}\right]_{\frac{1}{2}}+\left[\mathscr{A} \phi, R_{+}\right]_{\frac{1}{2}}\right)^{1)} .
$$

The inequality (3.2) was established by several authors (c.f.e.g., Koppelman-Pincus [5], J. Schwartz [14], Widom [15], Shamir [11] and for any dimensional case Shamir [13]). Now we set $u_{i}-v_{i}=w_{i}, \varphi_{j}-\psi_{j}=$ $B_{1 j}^{-} w_{1}+\left.B_{2 j}^{-} w_{2}\right|_{t=0}=\omega_{j}$ in the representation formulas (2. 15). Then it follows from Proposition 2. 1 that

$$
D^{\alpha} w_{i}(x, t)=\sum_{j=1}^{2 m} \int D^{\alpha} K_{i j}(x-y, t) \omega_{j}(y) d y,|\alpha| \geqq 2 m-1 .
$$

Put $l_{j}^{ \pm}=2 m-1-m_{j}^{ \pm}$. Then we obtain from (3.3) by integration by parts

$$
\begin{gathered}
D_{x}^{l^{+}}\left(B_{1 k}^{+} w_{1}+B_{2 k}^{+} w_{2}\right)(x, t) \\
=\sum_{j=1}^{2 m} \int_{-\infty}^{\infty}\left\{D_{x}^{l^{+}}\left[B_{1 k}^{+} K_{1 j}+B_{2 k}^{+} K_{2 j}\right](x-y, t)\right\} \cdot \\
D_{x}^{l-j}\left(B_{1 j}^{-} w_{1}+B_{2 j}^{-} w_{2}\right)(y, 0) d y .
\end{gathered}
$$

Let $t$ tend to zero in both sides of (3. 4). Then we have

$$
\begin{aligned}
& D_{x}^{l^{+}}\left(B_{1 k}^{+} w_{1}+B_{2 k}^{+} w_{2}\right)(x, 0) \\
&=\int_{-\infty}^{\infty} \sum_{j=1}^{2 m}\left(c_{k j} \mathscr{H}^{+}+d_{k j} \mathscr{H}^{-}\right) \cdot \\
& \quad D_{x}^{l j}\left[B_{1 j}^{-} w_{1}+B_{2 j}^{-} w_{2}\right](y, 0) d y,
\end{aligned}
$$

where $\left\{c_{k j}\right\},\left\{d_{k j}\right\}$ are two matrices with constant coefficients. Put $C=\left\{c_{k j}\right\}$ and $D=\left\{d_{k j}\right\}$. We make the following assumption.

Assumption 3.1. Two matrices $C, D$ are non singular and eigenvalues of $C^{-1} D$ do not lie on the negative real axis.

Then we have

Theorem 3.1. Under Assumption 3. 1, the mixed a priori estimates (3. 1) holds.

1) If $\phi=\left(\phi_{1}, \cdots, \phi_{2 m}\right)$, we set $\|\phi, \Omega\|_{s}=\Sigma\left\|\phi_{i}, \Omega\right\|_{s}$ and $[\phi, \Omega]_{s}=\sum\left[\phi_{i}, \Omega\right]_{s}$. 
Proof. We set

$$
\begin{aligned}
& \varphi_{j}(x)=D_{x}^{l \bar{j}}\left(B_{1 j}^{-} w_{1}+B_{2 j}^{-} w_{2}\right)(x, 0), \\
& \psi_{k}(x)=D_{x}^{l+}\left(B_{1 k}^{+} w_{1}+B_{2 k}^{+} w_{2}\right)(x, 0)
\end{aligned}
$$

and

$$
\varphi=\left(\varphi_{1}, \cdots, \varphi_{2 m}\right), \quad \psi=\left(\psi_{1}, \cdots, \psi_{2 m}\right) .
$$

We have by (3.5)

$$
\psi=\left(C \mathscr{H}^{+}+D \mathscr{H}^{-}\right) \varphi .
$$

Since $\varphi \in W^{\frac{1}{2}}(R)$ from (2.13), Proposition 3.1 is applicable to the equation (3. 6). Hence it follows that

$$
\begin{aligned}
& \sum_{j}\left\|B_{1 j}^{-} w_{1}+B_{2 j}^{-} w_{2}, R\right\|_{2 m-m_{j}-\frac{1}{2}} \\
& \leqq C \sum_{j, \pm}\left\|B_{1 j}^{+} w_{1}+B_{2 j}^{+} w_{2}, R_{+}\right\|_{2 m-m_{j}^{+}-\frac{1}{2}} .
\end{aligned}
$$

Since $w_{i}=u_{i}-v_{i}$, we see

$$
\left\|B_{1 j}^{ \pm} w_{1}+B_{2 j}^{ \pm} w_{2}, R_{ \pm}\right\|_{2 m-m_{j}^{ \pm}-\frac{1}{2}}
$$

$$
\begin{aligned}
\leqq \| B_{1 j}^{ \pm} u_{1} & +B_{2 j}^{ \pm} u_{2}, R_{ \pm} \|_{2 m-m_{j}^{ \pm}-\frac{1}{2}} \\
& +\left\|B_{1 j}^{ \pm} v_{1}+B_{2 j}^{ \pm} v_{2}, R_{ \pm}\right\|_{2 m-m_{\frac{j}{j}-\frac{1}{2}}}
\end{aligned}
$$

According to the well known result (c.f.e.g. [1], [8]) there exists a constant $C$ depending only on $k(\geqq 0)$ such that the following inequality holds:

$$
\|f, R\|_{k} \leqq C\left\|f, R_{+}^{2}\right\|_{k+\frac{1}{2}}
$$

for all $f \in C^{\infty}\left(\bar{R}_{+}^{2}\right)$.

Thus we see from (3.9)

$$
\begin{aligned}
\| B_{1 j}^{ \pm} v_{1} & +B_{2 j}^{ \pm} v_{2}, R_{ \pm} \|_{2 m-m \frac{ \pm}{j}-\frac{1}{2}} \\
& \leqq\left\|B_{1 j}^{ \pm} v_{1}+B_{2 j}^{ \pm} v_{2}, R\right\|_{2 m-m \frac{m}{j}-\frac{1}{2}} \\
& \leqq\left\|B_{1 j}^{ \pm} v_{1}+B_{2}^{ \pm} v_{2}, R_{ \pm}^{2}\right\|_{2 m-m_{j}^{ \pm}} \\
& \leqq C\left(\left\|v_{1}, R_{+}^{2}\right\|_{2 m}+\left\|v_{2}, R_{+}^{2}\right\|_{2 m}\right) .
\end{aligned}
$$

Using the inequalities (2. 16) and (3.9), we have

$$
\begin{aligned}
\| B_{1 j}^{ \pm} v_{1} & +B_{2 j}^{ \pm} v_{2}, R_{ \pm} \|_{2 m-m \frac{m}{j}-\frac{1}{2}} \\
& \leqq C\left(\left\|L_{1} u_{1}, R_{+}^{2}\right\|_{0}+\left\|L_{2} u_{2}, R_{+}^{2}\right\|_{0}\right) .
\end{aligned}
$$

On the other hand it follows from Proposition 2. 2 that 


$$
\begin{aligned}
\left\|u_{1}, R_{+}^{2}\right\|_{2 m} & +\left\|u_{2}, R_{+}^{2}\right\|_{2 m} \leqq C\left(\left\|L_{1} u_{1}, R_{+}^{2}\right\|_{0}\right. \\
& +\left\|L_{2} u_{2}, R_{+}^{2}\right\|_{0} \\
& +\sum_{j=1}^{2 m}\left\|B_{1 j}^{-} v_{1}+B_{2 j}^{-} v_{2}, R\right\|_{2 m-m_{\bar{j}}} \\
& +\sum_{j=1}^{2 m}\left\|B_{1_{j}}^{-} w_{1}+B_{2 j}^{-} w_{2}, R\right\|_{2 m-m_{j}} \\
& \left.+\left\|u_{1}, R_{+}^{2}\right\|_{0}+\left\|u_{2}, R_{+}^{2}\right\|_{0}\right) .
\end{aligned}
$$

Combining (3. 7), (3. 8), (3. 10) and (3.11), we obtain the proof of the theorem.

4. In this section we shall prove coerceive inequalities for a singular domain. Let $\mathscr{D}$ be an open disk with the center $O$ and radius $r$ which has an incision along the positive $x$ axis. We denote by $\Gamma_{1}, \Gamma_{2}$ the upper and lower boundary portions of the incision respectively. Let $\tilde{\mathscr{D}}$ be the closure of the subspace $\mathscr{D}$ in a manifold which distinguish between $\Gamma_{1}$ and $\Gamma_{2}$. Put $\tilde{C}_{0}^{\infty}(\mathscr{D})=\left\{u \in C^{\infty}(\tilde{\mathscr{D}}) \mid u=0\right.$ in a neighborhood of $|x|=0$ and $|x|=r\}$.

Let us consider an elliptic differential operator $L(D)$ of the form (2.1) and let $\left\{\tilde{B}_{i j}\right\}_{j=1}^{m}$ be a set of boundary operators on $\Gamma_{i}$ such that $\widetilde{B}_{i j}$ is homogeneous of degree $m_{j}(<2 m)$.

Set

$$
\begin{gathered}
L_{1}(D)=L(D), \quad L_{2}(D)=L\left(D_{x},-D_{t}\right), \\
B_{1 j}^{+}(D)=\widetilde{B}_{1 j}(D), \quad B_{2 j}^{+}(D)=\widetilde{B}_{2 j}\left(D_{x},-D_{t}\right) \\
B_{1 j}^{-}(D)=D_{t}^{j-1}, \quad B_{2 j}^{-}(D)=(-1)^{j} D_{t}^{j-1}, \\
j=1, \cdots, m .
\end{gathered}
$$

Then we can prove the following

Theorem 4. 1. If $\left\{L_{i}(D), B_{i j}^{ \pm}(D)\right\}$ of type (4. 1) satisfies Assumption 3. 1 and if $\left\{L_{i}(D), B_{i j}^{+}(D)\right\}$ satisfies the Complementing Condition, then there exists a constant $C$ such that

$$
\begin{aligned}
\|u, \mathscr{D}\|_{2 m} \leqq & C\left(\|L u, \mathscr{D}\|_{0}+\sum_{j=1}^{m}\left\|\tilde{B}_{1 j} u, \Gamma_{1}\right\|_{2 m-m_{j}-\frac{1}{2}}\right. \\
& +\sum_{j=1}^{m}\left\|\tilde{B}_{2 j} u, \Gamma_{2}\right\|_{2 m-m_{j}-\frac{1}{2}} \\
& \left.+\|u, \mathscr{D}\|_{0}\right)
\end{aligned}
$$


for all $u \in \widetilde{C}_{0}^{\infty}(\mathscr{D})$.

Proof. Put

$$
u_{1}(x, t)=u(x, t), \quad u_{2}(x, t)=u(x,-t), \quad t>0 .
$$

Then we easily see

$$
\begin{aligned}
& B_{1 j}^{-} u_{1}+B_{2 j}^{-} u_{2}=0, \quad t=0, \\
& \left.\tilde{B}_{1 j} u\right|_{\Gamma_{1}}=\left.B_{1 j}^{+} u_{1}\right|_{t=0}
\end{aligned}
$$

and

$$
\left.\widetilde{B}_{2 j} u\right|_{\Gamma_{2}}=\left.B_{2 j}^{+} u_{2}\right|_{t=0} \text {. }
$$

Thus it is sufficient to prove that

$$
\begin{aligned}
\left\|u_{1}, R_{+}^{2}\right\|_{2 m} & +\left\|u_{2}, R_{+}^{2}\right\|_{2 m} \leqq C\left(\left\|L_{1} u_{1}, R_{+}^{2}\right\|_{0}+\left\|L_{2} u_{2}, R_{+}^{2}\right\|_{0}\right. \\
& +\sum_{j=1}^{2 m}\left\|B_{1 j}^{-} u_{1}+B_{2 j}^{-} u, R_{-}\right\|_{2 m-m \bar{j}} \\
& +\sum_{j=1}^{2 m}\left\|B_{1 j}^{+} u_{1}+B_{2 j}^{+} u_{2}, R_{+}\right\|_{2 m-m_{j}^{+}} \\
& \left.+\left\|u_{1}, R_{+}^{2}\right\|_{0}+\left\|u_{2}, R_{+}^{2}\right\|_{0}\right) .
\end{aligned}
$$

This inequality follows from Theorem 3. 1. So, the proof of Theorem 4.1 is obtained.

Let $\Omega$ be a singular domain in our sense. Denote by $\tilde{C}^{\infty}(\bar{\Omega})$ a set of functions which are $C^{\infty}$ in $\bar{\Omega}$ and vanish near the endpoints of each boundary portion. We consider an elliptic operator of order $2 m$ in the form

$$
L(P, D)=\sum_{|\alpha| \leqq 2 m} a_{凶}(x, t) D_{x}^{\alpha_{1}} D_{t}^{\alpha_{2}}, \quad a_{\alpha}(x, t) \in C^{\infty}(\bar{\Omega}) .
$$

On each boundary portion $\Gamma_{i}$ there are defined $m$ partial differential operators

$$
B_{i j}(P, D)=\sum_{|\alpha| \leqq m_{i j}} b_{i j_{\alpha}}(x, t) D_{x}^{x_{1}} D_{t}^{\alpha}, \quad j=1, \cdots, m,
$$

where $m_{i j}<2 m$ and the coefficients are in $C^{\infty}\left(\Gamma_{i}\right)$.

We make the following assumption.

Assumption 4. 1. We assume that the boundary set $\left\{B_{i j}(P, D)\right\}_{j=1}^{m}$ is normal in the sense of [8] and satisfies the Complementing Condition. 
Let $P_{0}$ be an endpoint of a boundary portion $\Gamma_{i}$. For a real vector $\tau$ tangent to $\Gamma_{i}$ at $P_{0}$ and a real vector $\nu$ normal to $\Gamma_{i}$ at $P_{0}$, we rewrite the operators $L\left(P_{0}, D\right), B_{i j}\left(P_{0}, D\right)$ of type (4.3), (4.4) in the form

$$
\begin{aligned}
L\left(P_{0}, D\right) & =L\left(P_{0}, D_{x}, D_{t}\right) \\
= & \tilde{L}\left(P_{0}, D_{\tau}, D_{\nu}\right)=\tilde{L}\left(P_{0}, \tilde{D}\right), \\
B_{i j}\left(P_{0}, D\right) & =B_{i j}\left(P_{0}, D_{x}, D_{t}\right) \\
= & \widetilde{B}_{i j}\left(P_{0}, D_{\tau}, D_{\nu}\right)=\widetilde{B}_{i j}\left(P_{0}, \tilde{D}\right), \\
& 1 \leqq j \leqq m,
\end{aligned}
$$

where $D_{\tau}=\frac{\partial}{\partial \tau}$ and $D_{\nu}=\frac{\partial}{\partial \nu}$. Then we have the following

Theorem 4.2. Under Assumption 4. 1, consider operators $L(P, D), B_{i j}(P, D)$ of type (4. 3), (4. 4) in a singular domain $\Omega$. Suppose that $\tilde{L}\left(P_{0}, \tilde{D}\right), \widetilde{B}_{i j}\left(P_{0}, \tilde{D}\right)$ of the form (4.5) satisfy Assumption 3.1 for each endpoint $P_{0}$ of boundary portions. Then there is a constant $C$ depending only on $L(P, D), B_{i j}(P, D)$ and such that

$$
\begin{gathered}
\|u, \Omega\|_{2 m} \leqq C\left(\|L(P, D) u, \Omega\|_{0}\right. \\
+\sum_{i, j}\left\|B_{i j}(P, D) u, \Gamma_{i}\right\|_{2 m-m_{j}-\frac{1}{2}} \\
\left.+\|u, \Omega\|_{0}\right)
\end{gathered}
$$

for all $u \in \tilde{C}^{\infty}(\bar{\Omega})$.

Proof. The passage from the equations with constant coefficients in a half space to the estimate (4.6) is performed in a familiar method based on a partition of unity (c.f.e.g. $[4,8,9,10]$ ). Thus we shall show (4.6) only in a neighborhood of the endpoints of each $\Gamma_{i}$.

Let $P_{0}$ be an endpoint of $\Gamma_{i}$. From our definition of singular domains, we can take a sufficiently small neighborhood $U\left(P_{0}\right)$ of $P_{0}$ such that $U\left(P_{0}\right)$ can be mapped in a one-to-one $C^{\infty}$ way into an open disk $\mathscr{D}$ which has an incision along the positive $x$ axis. By applying Theorem 3. 1, it follows that

$$
\begin{aligned}
&\left\|u, U\left(P_{0}\right) \cap \Omega\right\|_{2 m} \leqq C\left(\left\|L\left(P_{0}, D\right) u, U\left(P_{0}\right) \cap \Omega\right\|_{0}\right. \\
&+\sum_{j}\left\|B_{i_{1} j}\left(P_{0}, D\right) u, \Gamma_{i_{1}}\right\|_{2 m-m_{j}-\frac{1}{2}} \\
&+\sum_{j}\left\|B_{i_{2} j}\left(P_{0}, D\right) u, \Gamma_{i_{2}}\right\|_{2 m-m_{j}-\frac{1}{2}}+\left\|u, U\left(P_{0}\right) \cap \Omega\right\|_{0}
\end{aligned}
$$

for all $u \in \tilde{C}_{0}^{\infty}\left(U\left(P_{0}\right) \cap \Omega\right)$. Here $\tilde{C}_{0}^{\infty}\left(U\left(P_{0}\right)_{n} \Omega\right)=\left\{u \in C^{\infty}\left(U\left(P_{0}\right) \cap \Omega\right) \mid u=0\right.$ in a neighborhood of $P_{0}$ and $\left.\partial U\left(P_{0}\right)\right\}$. We see from (4.7) 


$$
\begin{aligned}
& \left\|u, U\left(P_{0}\right)_{\cap} \Omega\right\|_{2 m} \leqq C\left(\left\|L(P, D) u, U\left(P_{0}\right)_{\cap} \Omega\right\|_{0}\right. \\
& \quad+\sum_{j}\left\|B_{i_{1} j}(P, D) u, \Gamma_{i_{1}}\right\|_{2 m-m_{j}-\frac{1}{2}} \\
& \quad+\sum_{j}\left\|B_{i_{2} j}(P, D) u, \Gamma_{i_{2}}\right\|_{2 m-m_{j}-\frac{1}{2}} \\
& \quad+\left\|\left(L\left(P_{0}, D\right)-L(P, D)\right) u, U\left(P_{0}\right) \cap \Omega\right\|_{0} \\
& \quad+\sum_{j}\left\|\left(B_{i_{1} j}\left(P_{0}, D\right)-B_{i_{1} j}(P, D)\right) u, \Gamma_{i_{1}}\right\|_{2 m-m_{j}-\frac{1}{2}} \\
& \quad+\sum_{j}\left\|\left(B_{i_{2} j}\left(P_{0}, D\right)-B_{i_{2} j}(P, D)\right) u, \Gamma_{i_{2}}\right\|_{2 m-m_{j}-\frac{1}{2}} \\
& \left.\quad+\left\|u, U\left(P_{0}\right)_{\cap} \Omega\right\|_{0}\right) .
\end{aligned}
$$

By the well known interpolation method, we find a neighborhood $U\left(P_{0}\right)$ for a given $\varepsilon>0$ such that

$$
\begin{aligned}
& \left\|\left(L\left(P_{0}, D\right)-L(P, D)\right) u, U\left(P_{0}\right) \cap \Omega\right\|_{0} \\
& \leqq \varepsilon\left\|u, U\left(P_{0}\right) \cap \Omega\right\|_{2 m} \\
& \quad+C(\varepsilon)\left\|u, U\left(P_{0}\right)_{\cap} \Omega\right\|_{0}, \\
& \sum_{j}\left\|\left(B_{i_{k} j}\left(P_{0}, D\right)-B_{i_{k} j}(P, D)\right) u, \Gamma_{i_{k}}\right\|_{2 m-m_{j}-\frac{1}{2}} \\
& \leqq \varepsilon\left\|u, \Gamma_{i_{k}}\right\|_{2 m-\frac{1}{2}}+C(\varepsilon)\left\|u, \Gamma_{i_{k}}\right\|_{-\frac{1}{2}} \\
& \quad k=1,2 .
\end{aligned}
$$

By (3. 9) we see

$$
\begin{aligned}
& \sum_{k, j}\left\|\left(B_{i_{k} j}\left(P_{0}, D\right)-B_{i_{k}}(P, D)\right) u, \Gamma_{i_{k}}\right\|_{2 m-m_{j}-\frac{1}{2}} \\
& \quad \leqq C\left(\varepsilon\left\|u, U\left(P_{0}\right)_{\cap} \Omega\right\|_{2 m}+C(\varepsilon)\left\|u, U\left(P_{0}\right)_{\cap} \Omega\right\|_{0}\right) .
\end{aligned}
$$

Combining (4. 8), (4. 9) and (4. 10), we can find $U\left(P_{0}\right)$ such that

$$
\begin{aligned}
& \left\|u, U\left(P_{0}\right)_{\cap} \Omega\right\|_{2 m} \leqq C\left(\left\|L(P, D) u, U\left(P_{0}\right) \cap \Omega\right\|_{0}\right. \\
& \quad+\sum_{j}\left\|B_{i_{1} j}(P, D) u, \Gamma_{i_{1}}\right\|_{2 m-m_{j}-\frac{1}{2}} \\
& \quad+\sum_{j}\left\|B_{i_{2} j}(P, D) u, \Gamma_{i_{2}}\right\|_{2 m-m_{j}-\frac{1}{2}} \\
& \left.\quad+\left\|u, U\left(P_{0}\right) \cap \Omega\right\|_{0}\right)
\end{aligned}
$$

for all $u \in \widetilde{C}_{0}^{\infty}\left(U\left(P_{0}\right)_{\cap} \Omega\right)$. This inequality means that (4.6) holds in a neighborhood of the endpoints of $\Gamma_{i}$. The proof is thus complete.

5. Let us consider a set of partial differential operators $\{L(P, D)$, $\left.B_{i j}(P, D)\right\}$ of type (4. 3), (4. 4) in a singular domain $\Omega$. Throughout this section we assume that the set of boundary operators $\left\{B_{i j}(P, D)\right\}$ satisfies Assumption 4. 1. In this section we shall prove the alternative theorem 
for elliptic boundary value problems $\Pi\left(L, f, B_{i j}\right)$ in a singular domain. Our method is essentially along the lines of Schechter $[8,9,10]$. We denote by $\{S\}$ a set of all endpoints of boundary portion $\Gamma_{i}$.

Lemma 5. 1. There exists another boundary set $\left\{B_{i j}^{\prime}(P, D)\right\}$ satisfying Assumption 4.1 such that if $u \in C^{\infty}(\bar{\Omega}-\{S\})$ and if

$$
\left(u, L^{*} v\right)=(L u, v)
$$

for all $v \in \widetilde{C}^{\infty}(\bar{\Omega})$ satisfying $B_{i j}^{\prime} v=0$ on $\Gamma_{i}$, then $B_{i j} u=0$ on $\Gamma_{i}$.

The set $\left\{B_{i j}^{\prime}\right\}$ is called adjoint to $\left\{B_{i j}\right\}$ relative to $L$. The proof of Lemma 5.1 can be obtained in a quite similar manner to the proof developed by Aronszajn-Milgram [3] and Schechter [8] for regular domains. By a solution of the problem $\Pi\left(L, f, B_{i j}\right)$ we shall mean a function $u$ such that $u \in C^{\infty}(\bar{\Omega}-\{S\})_{\cap} L^{2}(\Omega)$ and such that

$$
L u=f \text { in } \Omega, \quad B_{i j} u=0 \text { on } \Gamma_{i}, \quad j=1, \cdots, m_{i j} .
$$

Theorem 5. 1. Let $\left\{L(P, D), B_{i j}(P, D)\right\}$ be a set of operators of type (4. 3), (4. 4) in a singular domain $\Omega$. Assume that the set of adjoint operators $\left\{L^{*}\left(P_{0}, D\right)\right.$, $\left.B_{i j}^{\prime}\left(P_{0}, D\right)\right\}$ satisfies Assumption 3. 1 for each endpoint $P_{0}$ of boundary portions. Then the boundary value problem $\Pi\left(L, f, B_{i j}\right)$ has a solution if the only solution of $\Pi\left(L^{*}, 0, B_{i j}^{\prime}\right)$ is $u=0$.

In the last section we shall give some example for Theorem 5. 1.

Proof. We proceed essentially the lines of Schechter $[9,10]$. Let $\tilde{H}(\Omega)$ be the completion of $\tilde{C}^{\infty}(\bar{\Omega})$ with respect to the norm

$$
\|u\|^{2}=\|u, \Omega\|_{2 m}^{2}+\sum_{i, j}\left\|B_{i j} u, \Gamma_{i}\right\|_{2 m-m}^{2} .
$$

It is easily verified that $\tilde{H}(\Omega)$ is a Hilbert space and is a subspace of $W^{2^{m}}(\Omega)$. We also set

$$
[u, v]=\iint_{\Omega} L^{*} u \overline{L^{*}} v d x d t+\sum_{i, j}\left(B_{i j}^{\prime} u, B_{i j}^{\prime} v\right)_{2 m-m_{i j, \Gamma_{i}}}
$$

for all $u, v \in \tilde{C}^{\infty}(\Omega)^{1)}$. Then we can see from Theorem 4.2 that $[u, v]$ is defined for $u, v \in \tilde{H}(\Omega)$ and that there is a positive constant $c$ such that

$$
c^{-1}\|u\|_{2 m}^{2} \leqq[u, u]+\|u\|_{0}^{2} \leqq c\|u\|_{2 m}^{2}
$$

for all $u \in \tilde{H}(\Omega)$. For simplicity we denote $\|u, \Omega\|_{k}$ by $\|u\|_{k}$.

1) Boundary inner products are defined by a partition of unity and Fourier transformation (see e.g. [8]). 
Now we can prove that there is a positive constant $c$ such that

$$
c^{-1}\|u\|_{2 m}^{2} \leqq[u, u] \leqq c\|u\|_{2 m}^{2}
$$

for all $u \in \tilde{H}(\Omega)$. Assume that the estimate (5.2) does not hold. Then there is a sequence $\left\{u_{n}\right\}$ belonging to $\tilde{H}(\Omega)$ such that $n^{-1}\left\|u_{n}\right\|_{2 m}^{2} \geqq\left[u_{n}, u_{n}\right]$.

If we put $v_{n}=u_{n} /\left\|u_{n}\right\|_{2 m}$, it follows that

$$
\left\|v_{n}\right\|_{2 m}=1, \quad v_{n} \in \tilde{H}(\Omega)
$$

and

$$
\left[v_{n}, v_{n}\right] \rightarrow 0 \quad(n \rightarrow \infty) .
$$

Applying Rellich's lemma to (5.3), we have a subsequence (which is also denoted by $\left\{v_{n}\right\}$ for the brevity) such that

$$
\left\|v_{n}-v\right\|_{0} \rightarrow 0 \quad(n \rightarrow \infty) .
$$

Now it follows from (5. 1) that

$$
\begin{gathered}
c^{-1}\left\|v_{n}-v_{n},\right\|_{2 m}^{2} \leqq \\
\leqq \\
\leqq \\
{\left[v_{n}, v_{n}\right]+\left[v_{n}, v_{n}, v_{n}\right]-\left[v_{n}, v_{n}\right]} \\
-\left[v_{n}, v_{n}\right]+\left\|v_{n}-v_{n},\right\|_{0}^{2} .
\end{gathered}
$$

By Schwarz inequality

$$
\left[v_{n}, v_{n}\right] \leqq\left[v_{n}, v_{n}\right]^{\frac{1}{3}}\left[v_{n}, v_{n}\right]^{\frac{1}{2}} .
$$

Combining (5. 4) (5.7), we see

$$
v_{n} \rightarrow v \text { in } W^{2 m}(\Omega) \text {. }
$$

Hence $[v, v]=\lim \left[v_{n}, v_{n}\right]=0$. This implies that $L^{*} v=0$ in $\Omega$ and $B_{i j}^{\prime} v={ }^{\prime} 0$ on $\Gamma_{i}$ in the weak sense. Applying the regularity theorem, we see that $v \in C^{\infty}(\bar{\Omega}-\{S\})_{\cap} L^{2}(\Omega)$. From our assumptions this means that $v=0$ in $\Omega$. On the other hand $\|v\|_{0}=\lim _{n \rightarrow \infty}\left\|v_{n}\right\|=1$. It is a contradiction. Thus (5.2) holds. That is, there is a constant $c>0$ such that

$$
\begin{aligned}
& |[u, v]| \leqq c\|u\|_{2 m}\|v\|_{2 m}, \\
& |[u, u]| \geqq c^{-1}\|u\|_{2 m}^{2}
\end{aligned}
$$

for all $u, v \in \widetilde{H}(\Omega)$. For a given function $f \in C^{\infty}(\bar{\Omega})$, the $L^{2}$ inner product $(f, v)$ is a bounded linear functional in $W^{2 m}(\Omega)$. Hence there is a function $g \in \tilde{H}(\Omega)$ such that 


$$
[g, v]=(f, v)
$$

for all $v \in \tilde{H}(\Omega)$ (c.f. [6]). If $v \in C_{0}^{\infty}(\Omega)$, (5. 8) implies

$$
\left(L^{*} g, L^{*} v\right)=(f, v) \text {. }
$$

Putting $L^{*} g=u$, we see

$$
\left(u, L^{*} v\right)=(f, v), \quad v \in C_{0}^{\infty}(\Omega) .
$$

Hence, $L u=f$ in $\Omega$ and $u \in C^{\infty}(\Omega)$. If we choose $v$ such as $v \in \tilde{C}^{\infty}(\bar{\Omega})$ and $B_{i j}^{\prime} v=0$ on $\Gamma_{i}$, then we see $u \in C^{\infty}(\bar{\Omega}-\{S\})$ by the regularity theorem. Thus we obtain the proof by Lemma 5.1

REMARK. When each $\Gamma_{i}$ is a closed smooth curve, N. Ikebe [4] has given the existence of solutions $C^{2^{m+\alpha}}(\bar{\Omega})(\alpha>0)$.

6. In this section we shall give some example for Theorem 5. 1. It is sufficient to give some example such that Assumption 3.1 holds. Let $\mathscr{D}$ be the disk defined in the beginning of section 4. We consider the Laplace operator $L(D)=\Delta$. Then the operators defined in (4.1) are of the form

$$
\begin{gathered}
L_{1}(D)=L_{2}(D)=\Delta \\
\left(\begin{array}{cc}
B_{11}^{-}(D) & B_{21}^{-}(D) \\
B_{12}^{-}(D) & B_{22}^{-}(D)
\end{array}\right)=\left(\begin{array}{cc}
1 & -1 \\
\frac{\partial}{\partial t} & \frac{\partial}{\partial t}
\end{array}\right) .
\end{gathered}
$$

Let us consider the boundary value problem (2.4) in $t \geqq 0$. That is,

$$
\begin{aligned}
& \Delta u_{1}=0, \quad \Delta u_{2}=0, \quad t \geqq 0, \\
& B_{11}^{-} u_{1}+B_{21}^{-} u_{2}=\varphi_{1}, \\
& B_{12}^{-} u_{1}+B_{22}^{-} u_{2}=\varphi_{2}, \quad t=0 .
\end{aligned}
$$

Then we see by direct calculation that the kernels in (2.10) are of the form

$$
\begin{aligned}
& \left(\begin{array}{rr}
R_{11}^{-}(x, t, \pm 1) & R_{21}^{-}(x, t, \pm 1) \\
R_{12}^{-}(x, t, \pm 1) & R_{22}^{-}(x, t, \pm 1)
\end{array}\right) \\
& =\left(\begin{array}{rr}
-2 G^{(2)}( \pm x+i t) & 2 G^{(2)}( \pm x+i t) \\
2 i G^{(2)}( \pm x+i t) & 2 i G^{(2)}( \pm x+i t)
\end{array}\right) .
\end{aligned}
$$


Hence by (2. 9), the Poisson kernels for the problem (6. 1) are of the following form:

$$
\left(\begin{array}{ll}
K_{11} & K_{21} \\
K_{12} & K_{22}
\end{array}\right)=\left(\begin{array}{cc}
c_{1}\left(z^{-1}-\bar{z}^{-1}\right) & c_{1}^{\prime}\left(z^{-1}-\bar{z}^{-1}\right) \\
c_{2}\left(\log z^{-1}+\log \left(-\bar{z}^{-1}\right)\right) & c_{2}\left(\log z+\log \left(-\bar{z}^{-1}\right)\right)
\end{array}\right)
$$

where $z=x+i y$ and $c_{i}$ are constants.

(I) Consider the boundary operators on the incision of $\mathscr{D}$ such as

$$
B_{1}(D) \equiv 1 \text { on } \Gamma_{1}, \quad B_{2}(D) \equiv-D_{t}+a D_{x} \text { on } \Gamma_{2} .
$$

Then from (4. 1)

$$
\left(\begin{array}{cc}
B_{11}^{+}(D) & B_{21}^{+}(D) \\
B_{12}^{+}(D) & B_{22}^{+}(D)
\end{array}\right)=\left(\begin{array}{cc}
1 & 0 \\
0 & \tau+a \xi
\end{array}\right)
$$

Thus by calculation of (3.4), the integral equation (3.6) is of the form

$$
\left(\begin{array}{l}
\psi_{1} \\
\psi_{1}
\end{array}\right)=\left(\begin{array}{cc}
-H^{+}+H^{-} & i H^{+}+i H^{-} \\
(i+a) H^{+}+(i-a) H^{-} & (a i-1) H^{+}+(a i+1) H^{-}
\end{array}\right)\left(\begin{array}{l}
\varphi_{1} \\
\varphi_{2}
\end{array}\right)
$$

that is, the matrices $C, D$ in Assumption 3.1 are

$$
C=\left(\begin{array}{cc}
-1 & i \\
i+a & a i-1
\end{array}\right), \quad D=\left(\begin{array}{cc}
1 & i \\
i-a & a i+1
\end{array}\right) \text {. }
$$

Hence we see

$$
C^{-1} D=\frac{i}{1-a i}\left(\begin{array}{cc}
a i-1 & -1 \\
-(i+a) & -1
\end{array}\right) .
$$

Thus we conclude that if $a$ is real, the boundary operators (6.2) satisfies Assumption 3. 1.

(II) Secondly we consider the boundary operators

$$
\begin{aligned}
& B_{1}(D) \equiv D_{t}+a D_{x} \text { on } \Gamma_{1}, \\
& B_{2}(D) \equiv-D_{t}+a D_{x} \text { on } \Gamma_{2} .
\end{aligned}
$$

Then proceeding similarly as in I), we see

$$
C^{-1} D=\left(\begin{array}{cc}
i+a & 1-a i \\
-(i+a) & 1-a i
\end{array}\right)^{-1}\left(\begin{array}{cc}
a-i, & a i+1 \\
i-a & a i+1
\end{array}\right) i+a\left(\begin{array}{cc}
1 & 0 \\
0 & -1
\end{array}\right) .
$$

If $a \neq 0$ and $a$ is not pure imaginary, we see that Assumption 3.1 is satisfied. 
When $a=0$, our assumption is not satisfied. But it is seen that the mixed a priori estimates (3. 1) hold from the relations

$$
I=\mathscr{H}^{+}-\mathscr{H}^{-}, \quad 2 \mathscr{H}^{-}=\mathscr{H}^{+}+\mathscr{H}^{-} \text {, }
$$

where $\mathscr{H}$ denotes Hilbert transform on the whole real line.

\section{BIBLIOGRAPHY}

[1] Agmon, S., Douglis, A., and Nirenberg, L., Estimates near the boundary for solutions of elliptic partial differential equations satisfying general boundary conditions I, Comm. Pure Appl. Math., 12, 1959, pp. 623-727.

[2] — II, ibid. 17, 1964, pp. 35-92.

[ 3 ] Aronszajn, N., and Milgram, A.N., Differential operators on Riemannian manifolds, Rend. Circ. Mat. Parlermo, Ser. 2, 2, pp. 1-61.

[4] Ikebe, N., On elliptic boundary value problems with discontinuous coefficients, Memoirs of the Fac. Sci. Kyushu Univ., Ser. A, 21, 1967, pp. 167-184.

[ 5 ] Koppelman, W., and Pincus, J.V., Spectral representations of finite Hilbert transformations, Math. Z., 71, 1959, pp. 399-407.

[6] Lax, P.D., and Milgram, A., Parabolic equations, Contributions to the Theory of Partial Differential Equations, Ann. of Math. Studies, Princeton University Press, 1954, pp. 167-190.

[ 7 ] Peetre, J., Mixed problems for higher order elliptic equations in two variables (I), Annali di Pisa, 15, 1961, pp. 337-353.

[ 8 ] Schechter, M., General boundary value problems for elliptic partial differential equations, Comm. Pure Appl. Math., 12, 1959, pp. 451-485.

[9] Schechter, M., Mixed boundary problems for general elliptic equations, Comm. Pure Appl. Math., 13, 1960, pp. 407-425.

[10] Schechter, M., A generalization of the problem of transmission, Annali della Scoula Norm. Sup. Pisa., Ser. 3, 13, 1960, pp. 207-236.

[11] Shamir, E., Reduced Hilbert transforms and singular integral equations, J. D'analyse Math., 12, 1964, pp. 277-305.

[12] Shamir, E., Mixed boundary value problems for elliptic equations in the plane. The $L^{p}$ theory, Annali di Pisa, 17, 1963, pp. 117-139.

[13] Shamir, E., Elliptic systems of singular integral operators. I. The half-space case, Trans. Amer. Math. Soc., 124, 1967, pp. 107-124.

[14] Schwartz, J., Some results on the spectra and spectral resolutions of a class of singular integral operators, Comm. Pure Appl. Math., 15, 1962, pp. 75-90.

[15] Widom, H., Singular integral equations in $L^{p}$, Trans. Amer. Math. Soc., 97, 1960, pp. 131-159.

\section{Nagoya University}

